\title{
Recuperação cardiopulmonar em atletas de Rugby em Cadeira de Rodas: efeito de nível competitivo e classificação funcional
}

\author{
Cardiopulmonary recovery in Wheelchair Rugby athletes: effects of \\ competitive level and functional classification
}

Alexssander de Souza Mello
Patrícia dos Santos Vigário $^{2}$
Jeter Pereira de Freitas $^{3}$
Thiago Lemos $^{4}$
Míriam Raquel Meira Mainenti

Endereço para correspondência

Míriam Raquel Meira Mainenti

Endereço: Avenida João Luis Alves s $/ \mathrm{n}^{\circ}$, sala dos instrutores -

Urca. Rio de Janeiro/RJ - Brasil. 22.291-090. Telefone: (21) 2586-2297.

Endereço eletrônico: miriam.mainenti@ hotmail.com

Bacharel em Educação Física, Escola de Educação Física do Exército (EsEFEx), Rio de Janeiro/RJ/Brasil.

alexcmrj@hotmail.com

Doutora, Programa de Pós Graduação em Ciências da Reabilitação, Centro Universitário Augusto Motta (UNISUAM), Rio de Janeiro/RJ/Brasil. patriciavigario@yahoo.com.br

Mestre, Programa de Pós Graduação em Ciências da Reabilitação, Centro Universitário Augusto Motta (UNISUAM), Rio de Janeiro/RJ/Brasil.

jeter.freitas@hotmail.com

Doutor, Programa de Pós Graduação em Ciências da Reabilitação, Centro Universitário Augusto Motta (UNISUAM), Rio de Janeiro/RJ/Brasil.

lemoscarvalho.thiago@gmail.com

Doutora, Escola de Educação Física do Exército (EsEFEx), Rio de Janeiro/RJ/Brasil.

miriam.mainenti@hotmail.com

\section{Resumo}

Introdução: o Rugby em Cadeira de Rodas (RCR) é uma modalidade com grande demanda energética. Objetivo: comparar a recuperação cardiopulmonar de atletas de RCR de elite internacional ( $E I, n=16$ ) e nacional ( $E N, n=06$ ), além de investigar a correlação entre a recuperação e a classificação funcional $(\mathrm{CF})$ dos atletas.

Métodos: o teste de esforço cardiopulmonar foi realizado em cicloergômetro para membros superiores, com análise dos gases expirados. Consumo de oxigênio $\left(\mathrm{VO}_{2}\right)$ e ventilação pulmonar (VE) foram avaliados no pico do esforço e na recuperação (primeiro, segundo e terceiro minutos).

Resultados: EI apresentou uma maior recuperação do $\mathrm{VO}_{2}$ e da VE. Correlações fortes e muito fortes foram encontradas entre $\mathrm{CF}$ e recuperação percentual de $\mathrm{VO}_{2}$ para $\mathrm{EN}$, enquanto que, no EI, as correlações foram fracas e regulares.

Conclusão: Atletas com maior volume de prática do RCR (EI) têm maior capacidade de recuperação cardiopulmonar. Sugere-se que o tempo de treinamento pode minimizar a influência da CF na capacidade de recuperação do atleta.

Descritores: Pessoas com deficiência. Quadriplegia. Consumo de oxigênio. Ventilação pulmonar.

\begin{abstract}
Introduction: Wheelchair Rugby (WR) is a sports modality with a high energetic demand. Objective: compare the cardiopulmonary recovery capacity of international (IE, $\mathrm{n}=16$ ) and national (NE, $\mathrm{n}=06$ ) elite $\mathrm{WR}$ athletes, and also verify the correlation between this capacity and the WR functional classification.

Methods: the cardiopulmonary exercise test was performed on a cycle ergometer (for upper limbs), with expired gas analysis. Oxygen consumption $\left(\mathrm{VO}_{2}\right)$ and pulmonary ventilation (VE) were analyzed at peak effort and during recovery (first, second and third minutes).

Results: The IE group presented a greater recovery for $\mathrm{VO}_{2}$ and VE. Strong and very strong correlations were found between functional classification and $\mathrm{VO}_{2}$ percentage recovery for NE group, but for IE, the correlations were only weak and regular ones. Conclusion: athletes with higher volume WR practice (IE) present better cardiopulmonary recovery capacity. The results for correlations suggest that time of sports practice possible minimize the influence of classification on recovery capacity of these athletes.
\end{abstract}

Keywords: Disabled Persons. Quadriplegia. Oxygen consumption. Pulmonary ventilation.

\section{Cite como \\ Vancouver}

Mello AS, Vigário PS, Freitas JP, Lemos T, Mainenti MRM. Recuperação cardiopulmonar em atletas de Rugby em Cadeira de Rodas: efeito de nível competitivo e classificação funcional. Conscientiae Saúde 2019 out./dez.;18(4):429-443. https://doi.org/10.5585/conssaude.v18n4.14963. 


\section{Introdução}

O Rugby em Cadeira de Rodas (RCR) surgiu em 1977, no Canadá, como um esporte coletivo para indivíduos com tetraplegia ou tetraequivalência ${ }^{1}$. São elegíveis para a prática do RCR pessoas com lesão medular em nível cervical, com sequelas de poliomielite, indivíduos com múltiplas amputações, dentre outros ${ }^{2}$. Por ser uma modalidade de intensidade de esforço variada, com quatro quartos de oito minutos, tanto a capacidade aeróbica quanto a anaeróbica são exigidas para resistir à duração de uma partida ${ }^{3-5}$. Para o acompanhamento da capacidade aeróbica, a avaliação dos atletas deve incluir uma análise do consumo de oxigênio em esforço, variável que melhor retrata a capacidade em questão ${ }^{6-7}$, inclusive em atletas com deficiência ${ }^{3,8}$.

Sabe-se que o consumo de oxigênio de pico, valor mais alto obtido em um teste de esforço progressivo ${ }^{9}$, e a capacidade pulmonar máxima encontram-se reduzidos em indivíduos com tetraplegia ${ }^{3,10,11}$. Justificativas para esses achados compreendem as alterações no sistema simpático, promovendo reduções na capacidade ventilatória, no débito cárdico e no retorno venoso. Esse último também sofre influência da permanência prolongada na posição sentada, que provoca acúmulo de sangue nos membros inferiores e consequentes alterações no fluxo sanguíneo ${ }^{11}$. Todo esse conjunto de modificações demonstra que, em situações de alta demanda metabólica, o organismo de um indivíduo com lesão medular dificilmente acompanhará o ritmo e a capacidade de indivíduos sem essa lesão ${ }^{12}$. Essa dificuldade de atender a situações de alta demanda metabólica sustenta a regra de não existir um número máximo de substituições de jogadores durante uma partida ${ }^{13}$. Dentre os objetivos da substituição de um jogador, está a recuperação cardiopulmonar, além do importante controle da temperatura corporal.

A recuperação de um esforço de alta intensidade cursa com alterações em diversos sistemas orgânicos para repor as fontes energéticas, remover os produtos do metabolismo e manter a homeostase. Nesse sentido, o sistema cardiopulmonar se mantém em atividade além dos níveis pré-esforço, com valores ainda aumentados para ventilação pulmonar, consumo de oxigênio, aporte sanguíneo local, dentre outros ${ }^{14}$. Quanto mais rápido ocorre essa recuperação, mais prontamente disponível o atleta estará para uma próxima jogada com, provavelmente, maior probabilidade de bom desempenho. Considerando o constante uso de momentos de recuperação em atletas de RCR, a capacidade de recuperação do sistema cardiopulmonar pode ser um importante parâmetro para avaliar a aptidão dos atletas dessa modalidade.

Apesar da literatura apresentar estudos avaliando a capacidade cardiopulmonar máxima em esforço em atletas de $\mathrm{RCR}^{3,15,16}$, não há pesquisas relacionadas ao comportamento 
cardiopulmonar durante a recuperação após um esforço máximo. Em modalidades esportivas convencionais, já foi mostrada uma associação positiva entre a capacidade de recuperação e o volume de treinamento ${ }^{17}$, no entanto, em esportes para pessoas com deficiência, essa relação ainda não está clara.

Uma particularidade de modalidades do programa paralímpico é a classificação funcional do atleta, que tem como objetivo tentar garantir a equidade dos participantes durante as competições ${ }^{2}$. De acordo com a funcionalidade muscular e a capacidade de realizar movimentos do RCR, cada atleta recebe uma pontuação que varia de 0,5 (maior comprometimento, geralmente os defensores) a 3,5 pontos (menor comprometimento, geralmente os atacantes). Nesse sentido, essa é outra variável com potencial de influenciar a capacidade de recuperação do atleta, mas tal correlação, no RCR, não foi ainda verificada.

Dessa forma, o presente estudo teve como objetivo analisar a recuperação das variáveis cardiopulmonares após esforço máximo de atletas de RCR, comparando os valores obtidos de acordo com o nível competitivo: participantes de competições internacionais (EI - atletas de elite internacional) e participantes de competições nacionais (EN - atletas de elite nacional). Adicionalmente, o estudo procurou investigar se a classificação funcional se correlaciona com a velocidade dessa recuperação. A hipótese inicial do estudo é que atletas de elite internacional apresentam reduções mais expressivas nas variáveis cardiopulmonares que atletas de elite nacional. A outra hipótese é que atletas com menor classificação funcional apresentam menor magnitude de recuperação.

O conhecimento da capacidade de recuperação cardiopulmonar dos atletas de rugby em cadeira de rodas auxiliará a equipe técnica na preparação física e tomada de decisão durante as partidas. Em especial, tal informação subsidiará a identificação dos atletas com potencial de retornar à quadra, após uma substituição, em melhores condições de jogo. Caso seja identificado que atletas de maior expressão apresentam maiores magnitudes de recuperação das variáveis cardiopulmonares, esse pode ser um interessante parâmetro para acompanhamento periódico do preparador físico. Adicionalmente, rotinas poderão ser revistas para aumentar o volume de treinamento e facilitar diversas adaptações nos sistemas orgânicos envolvidos na recuperação pós-esforço de alta intensidade. Por fim, a correlação da recuperação cardiopulmonar com a classificação funcional trará a informação do que pode ser esperado de atletas de diferentes níveis de comprometimento motor, informando à equipe técnica da necessidade de um maior 
ou menor tempo de recuperação (em treinos e jogos) de acordo com a classificação funcional do atleta.

\section{Material e métodos}

\section{Estudo e amostra}

Um estudo de natureza seccional foi conduzido com atletas da Seleção Brasileira de RCR que competiram nos Jogos Parapanamericanos de 2015 (atletas de elite internacional; EI) e atletas de uma equipe da primeira divisão do campeonato Brasileiro de RCR (atletas de elite nacional; EN), amostra de conveniência.

Os critérios de inclusão adotados foram: homens; idade maior ou igual a 18 anos; apresentar lesão medular a nível cervical; classificação funcional emitida pela Associação Brasileira de Rugby em Cadeira de Rodas (ABRC), nos casos de atletas classificados em campeonatos nacionais, ou na International Wheelchair Rugby Federation (IWRF), em atletas classificados em campeonatos internacionais; ter participado de competições de nível nacional e internacional (esse último, somente para os atletas do grupo EI) nos 12 meses prévios à realização do estudo. Como critério de exclusão, foi considerada a presença de dor ou lesão que pudesse impedir ou interferir na realização do teste de esforço cardiopulmonar.

\section{Aspectos éticos}

O estudo foi submetido e aprovado pelo sistema CEP-CONEP (CAAE: 17691113.1.0000.5235) e todos os participantes assinaram o Termo de Consentimento Livre e Esclarecido para a participação no estudo.

\section{Avaliações realizadas}

Os atletas foram submetidos a um teste cardiopulmonar de esforço máximo em um cicloergômetro para membros superiores (Excite, TechnoGym, Itália). Foi adotado um protocolo incremental de intensidade, com carga inicial de $20 \mathrm{~W}$ e aumentos de $2 \mathrm{~W}$ (atletas com classificação funcional de 0,5 a 1,5) ou 5W (atletas com classificação funcional de 2,0 a 3,5) a cada minuto. Essa diferenciação de incremento visou propiciar condições similares aos dois grupos em compensação às suas restrições motoras, de forma a atingirem o pico do esforço 
entre 8 e 12 minutos, conforme recomendado pela American Heart Association ${ }^{6}$. A recuperação pós-esforço máximo foi feita durante três minutos, de forma ativa e sem carga.

Antes do início do teste, foi verificado se todas as solicitações pré-teste foram cumpridas: não realizar atividade física extenuante; não ingerir bebidas alcoólicas ou cafeína no dia ou na noite anterior; e não fumar nas últimas quatro horas antes do teste.

A análise das trocas gasosas foi feita com o uso de um analisador metabólico de gases (VO2000, Medical Graphics; USA) e, para a medida da vazão respiratória, foi utilizado um pneumotacógrafo para fluxo médio (Medical Graphics, EUA). Os dados de vazão respiratória e frações expiradas de $\mathrm{O}_{2}$ e $\mathrm{CO}_{2}$ foram medidos a cada respiração, sendo armazenados como média de cada três respirações. As variáveis cardiopulmonares calculadas a partir desses valores utilizadas no presente estudo foram: consumo de oxigênio - $\mathrm{VO}_{2}\left(\mathrm{~mL} \mathrm{~kg}^{-1} \cdot \mathrm{min}^{-1}\right)$ e ventilação pulmonar - VE (L.min $\left.{ }^{-1}\right)$. Tais variáveis foram avaliadas no pico do esforço e na recuperação (primeiro, segundo e terceiro minutos).

O teste teve característica sintoma limitante, sendo interrompido por solicitação do avaliado ou no aparecimento de algum critério de interrupção, de acordo com o American College of Sports and Medicine ${ }^{18}$.

As variáveis de caracterização da amostra, incluindo tempo de lesão, tempo de prática da modalidade e volume semanal de treinamento, foram obtidas a partir de uma anamnese realizada antes do teste de esforço cardiopulmonar. A classificação funcional foi confirmada pelo documento emitido pela Associação Brasileira de Rugby em Cadeira de Rodas (ABRC), nos casos de atletas classificados em campeonatos nacionais, ou pela International Wheelchair Rugby Federation (IWRF), em atletas classificados em campeonatos internacionais.

\section{Tratamento estatístico}

Foram calculadas as variações do $\mathrm{VO}_{2}$ e da $\mathrm{VE}$ do pico para cada minuto de recuperação, em valores absolutos e percentuais (o quanto foi reduzido em relação ao valor de pico). Como as variáveis obedeceram a uma distribuição normal (avaliado pelo teste de Shapiro Wilk), foram adotadas estratégias paramétricas para apresentação e análise dos dados. Para caracterização da amostra, as variáveis numéricas foram apresentadas como média e desvio padrão, e as categóricas com frequência absoluta e relativa (\%).

Foram comparados os valores obtidos entre as duas equipes, EI e EN: para dados numéricos, foi utilizado o teste T-Student para amostras independentes, acompanhado do 
respectivo tamanho do efeito ( $d$ de Cohen, calculado como a diferença entre as médias dividida pelo desvio padrão agrupado); o teste de Fisher foi aplicado aos dados categóricos. Adicionalmente, foram feitas análises de correlação do comportamento da recuperação cardiopulmonar com o volume semanal de treinamento (com toda a amostra) e com a classificação funcional (com toda a amostra, bem como em cada nível competitivo) pelo cálculo dos coeficientes de correlação de Pearson. Todas as análises foram feitas no Programa SPSS (versão 13.0, EUA) e foram considerados significativos os valores de $\mathrm{p}<0,05$. A classificação para o coeficiente de correlação $(r)$ foi: muito forte para valores de $r \geq 0,90$; forte para $r$ entre 0,60 (inclusive) e 0,90; regular para $r$ entre 0,30 (inclusive) e 0,60; e fraca entre 0 e $0,30^{19}$.

\section{Resultados}

Foram incluídos no estudo 22 atletas de RCR, com idade $=31,27 \pm 5,82$ anos, massa corporal total $=70,25 \pm 9,75 \mathrm{~kg}$ e estatura $=1,78 \pm 0,07 \mathrm{~m}$. Cerca de $64,0 \%$ tinham a $\mathrm{CF}$ maior ou igual a 2 pontos (ponto alto; mais funcionais) e 36,0\% até 1,5 pontos inclusive (ponto baixo; menos funcionais). O tempo médio de prática da modalidade foi de 4,83 $\pm 2,52$ anos e o volume semanal de treinamento de $16,36 \pm 14,5$ horas. O tempo de lesão medular foi de 8,96 $\pm 4,15$ anos, sendo 45,4\% em C6-C7; 9,1\% em C5-C7; 18,2\% em C7; e 27,3\% em C5-C6.

Considerando o nível competitivo, os grupos $\operatorname{EN}(n=06)$ e EI $(n=16)$ foram similares no que diz respeito às variáveis antropométricas, demográficas e relativas à lesão. Foram observadas diferenças estatisticamente significativas entre os grupos nas variáveis tempo de prática do $\mathrm{RCR}(\mathrm{p}=0,038)$ e volume semanal $(\mathrm{p}=0,037)$, sendo maiores no grupo $\mathrm{EI}$, conforme esperado (Tabela 1). 
Tabela 1 - Variáveis antropométricas, demográficas, de treinamento e nível de lesão dos atletas avaliados, de acordo com o nível competitivo

\begin{tabular}{lcc}
\hline Variáveis & EN (n=06) & EI (n=16) \\
\hline Idade (anos) & $35,17 \pm 7,47$ & $29,81 \pm 4,53$ \\
MCT (kg) & $71,48 \pm 11,55$ & $69,79 \pm 9,36$ \\
Estatura (m) & $1,79 \pm 0,05$ & $1,78 \pm 0,08$ \\
Tempo de Lesão (anos) & $8,33 \pm 5,57$ & $9,19 \pm 3,67$ \\
Tempo de RCR (anos) & $3,04 \pm 2,72$ & $5,50 \pm 2,16^{*}$ \\
Volume semanal (horas) & $6,00 \pm 0,00$ & $20,25 \pm 15,34^{*}$ \\
Classificação Funcional; n(\%) & & $7(43,8)$ \\
0,5 a 1,5 & $1(16,7)$ & $9(56,2)$ \\
2,0 a 3,5 & $5(83,3)$ & $7(44)$ \\
Nível de lesão; n(\%) & & $2(13)$ \\
C6-C7 & $3(50)$ & $3(19)$ \\
C5-C7 & $0(0)$ & $4(25)$ \\
C7 & $1(17)$ & $2(33)$ \\
C5-C6 & &
\end{tabular}

Dados apresentados como média \pm desvio padrão para variáveis numéricas e frequência absoluta (frequência relativa) para as variáveis categóricas. Comparações entre grupos realizada pelo Teste T-Student para variáveis numéricas e teste de Fisher para as variáveis categóricas. EN - Grupo de atletas de elite nacional; EI - Grupo de atletas de elite internacional; MCT - Massa Corporal Total; CF - Classificação Funcional; RCR - Rugby em Cadeira de Rodas; $\mathrm{C}$ - vértebra cervical. $* \mathrm{p}<0,05$.

Fonte: Os autores.

Os valores de pico de $\mathrm{VO}_{2}$ foram maiores no grupo $\mathrm{EI}$ em comparação ao grupo EN: $27,27 \pm 6,62$ vs. $15,09 \pm 6,04 \mathrm{ml} \cdot \mathrm{kg}^{-1} \mathrm{~min}^{-1}(\mathrm{p}=0,001 ; d=-1,880)$. O mesmo foi observado para a VE: $54,92 \pm 13,64$ vs. 38,42 $\pm 10,63 \mathrm{~L} / \mathrm{min}(\mathrm{p}=0,015 ; d=-1,274)$. Os grupos apresentaram curvas de recuperação de $\mathrm{VE}$ e $\mathrm{VO}_{2}$ distintas pós-esforço, tendo sido observados melhores resultados para os atletas EI (Figuras 1 e 2). 
Figura 1 - Variação de consumo de oxigênio no $1^{\circ}, 2^{\circ}$ e $3^{\circ}$ minutos

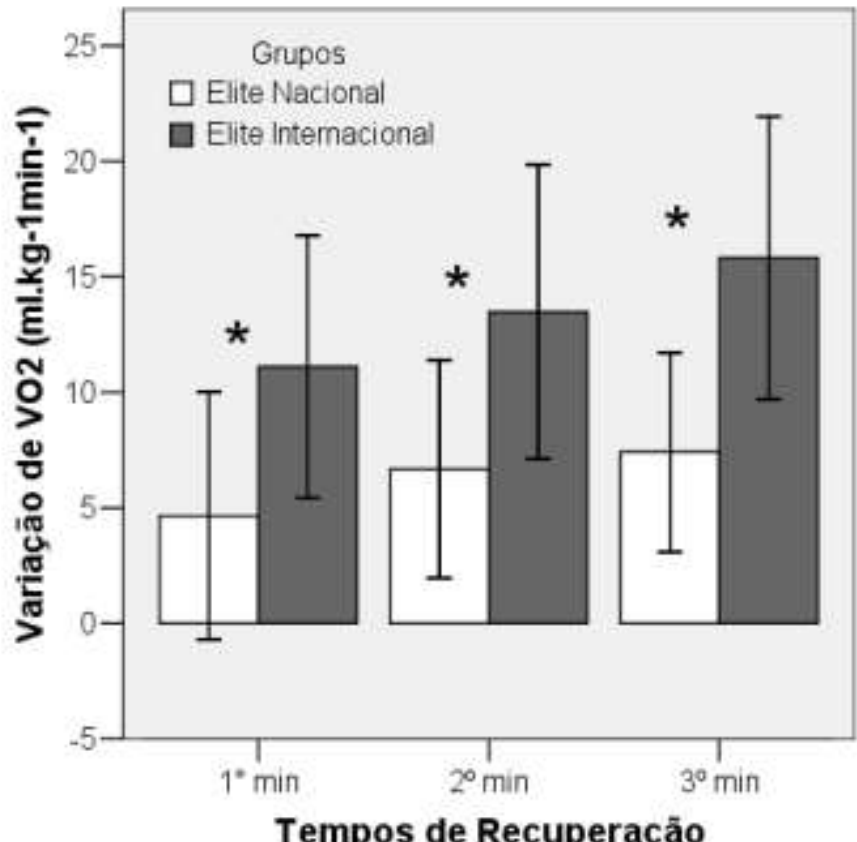

Gráfico de barras apresentando média e desvio padrão da variação de $\mathrm{VO} 2$ (ml.kg1min-1) nos três minutos de recuperação (d de Cohen -1,156; -1,135; $-1,471$, respectivamente para os minutos $1^{\circ}, 2^{\circ}$ e $3^{\circ}$ ) analisados para os grupos EN (atletas de elite nacional) e EI (atletas de elite internacional). $* \mathrm{p}<0,05$ (teste T-Student para amostras independentes).

Fonte: Os autores.

Figura 2 - Variação da ventilação pulmonar no $1^{\circ}, 2^{\circ}$ e $3^{\circ}$ minutos

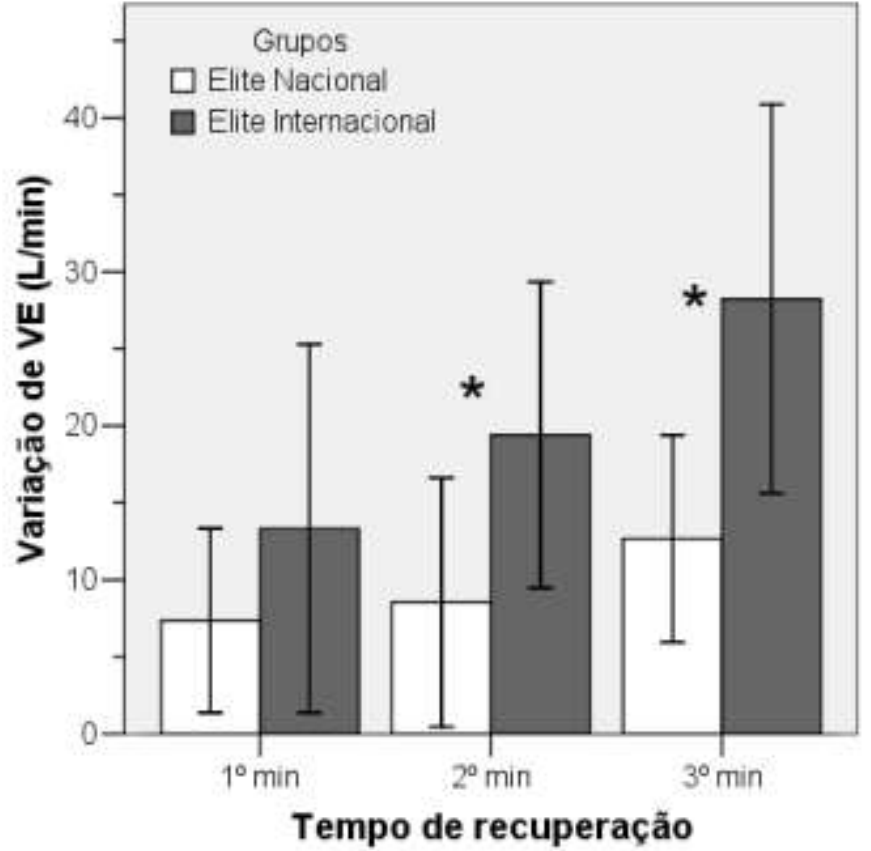

Gráfico de barras apresentando média e desvio padrão da variação de $\mathrm{VE}$ (L/min) nos três minutos de recuperação (d de Cohen 0,$555 ;-1,143$ e $-1,359$, respectivamente para os minutos $1^{\circ}, 2^{\circ}$ e $3^{\circ}$ ) analisados para os grupos EN (atletas de elite nacional) e EI (atletas de elite internacional). ${ }^{*} \mathrm{p}<0,05$ (teste $\mathrm{T}$-Student para amostras independentes).

Fonte: Os autores. 
Quando analisados em níveis relativos (percentual de variação em relação ao pico), foi observado um comportamento similar ao observado na análise sobre os valores absolutos, isto é, maiores valores no EI. Apesar da diferença estatisticamente significativa não ter sido identificada em todos os minutos, os valores médios da variação percentual de $\mathrm{VO}_{2}$ e VE apontam para um desempenho pior no grupo EN (Tabela 2).

Tabela 2 - Recuperação do consumo de oxigênio e da ventilação pulmonar (\% em relação ao pico do esforço) dos atletas avaliados, de acordo com o nível competitivo

\begin{tabular}{lcccc}
\hline Variáveis & EN $(\mathbf{n}=\mathbf{6})$ & $\mathbf{E I}(\mathbf{n}=\mathbf{1 6})$ & Valor de $\mathbf{p}$ & d de Cohen \\
\hline $\mathrm{VO}_{2 \text { pico }}-\operatorname{Rec} 1(\%)$ & $20,23 \pm 36,82$ & $38,59 \pm 13,62$ & 0,095 & $-0,840$ \\
$\mathrm{VO}_{2 \text { pico }}-\operatorname{Rec} 2(\%)$ & $38,83 \pm 19,87$ & $47,68 \pm 14,18$ & 0,255 & $-0,560$ \\
$\mathrm{VO}_{2 \text { pico }}-\operatorname{Rec} 3(\%)$ & $45,21 \pm 14,54$ & $57,10 \pm 13,07$ & 0,080 & $-0,884$ \\
$\mathrm{VE}_{\text {pico }}-\operatorname{Rec} 1(\%)$ & $18,07 \pm 9,94$ & $21,90 \pm 16,27$ & 0,598 & $-0,256$ \\
$\mathrm{VE}_{\text {pico }}-\operatorname{Rec} 2(\%)$ & $20,03 \pm 13,54$ & $34,21 \pm 13,42$ & $0,040^{*}$ & $-1,054$ \\
$\mathrm{VE}_{\text {pico }}-\operatorname{Rec} 3(\%)$ & $34,18 \pm 9,14$ & $50,20 \pm 15,12$ & $0,026^{*}$ & $-1,155$ \\
\hline
\end{tabular}

Dados apresentados como média \pm desvio padrão.

$\mathrm{VO}_{2}$ - Consumo de oxigênio; VE - Ventilação pulmonar; Rec1, 2 e 3 - Recuperação nos três minutos subsequentes ao esforço; EN - atletas de elite nacional; EI - atletas de elite internacional. *p < 0,05 (Teste T-Student para amostras independentes).

Fonte: Os autores.

As correlações entre o volume semanal de treinamento e a recuperação cardiopulmonar foram, em sua maioria, positivas, com o coeficiente de correlação de Pearson variando de $-0,03$ a 0,53. Correlações em dois momentos apresentaram significância estatística, especificamente com a recuperação (absoluta) de $\mathrm{VO}_{2}$ : no $1^{\circ}$ minuto $(\mathrm{r}=0,53 ; \mathrm{p}=0,012)$ e no $2^{\circ}$ minuto $(\mathrm{r}=$ $0,50, p=0,017)$, mas ambas de força regular.

$\mathrm{Na}$ investigação da correlação entre a classificação funcional e a recuperação do consumo de oxigênio, considerando toda a amostra $(n=22)$, foi identificada uma correlação "regular" com a variação de $\mathrm{VO}_{2}$ somente no primeiro minuto, tanto em valores absolutos $(\mathrm{r}=0,456, \mathrm{p}=0,033)$ quanto em percentual $(\mathrm{r}=0,465, \mathrm{p}=0,029)$. Não foram identificados coeficientes de correlação maiores que esse nos minutos subsequentes, acompanhados de análise sem significância estatística. Considerando os subgrupos do estudo (correlações feitas separadamente), foram encontradas correlações importantes no EN para as variações percentuais de $\mathrm{VO}_{2}$ e a $\mathrm{CF}$, classificadas como "muito forte" no primeiro $(\mathrm{r}=0,929, \mathrm{p}=0,007)$ e no terceiro $(r=0,951, p=0,004)$ minutos, e "forte" no segundo minuto $(r=0,882, p=0,020)$. Para 
a variação absoluta, os coeficientes variaram de 0,69 a 0,74, porém sem significância estatística. O EI (n=16) demonstrou uma correlação "regular" da CF com as variações absolutas no primeiro minuto de recuperação ( $\mathrm{r}=0,577, \mathrm{p}=0,019)$, no entanto, em níveis relativos, nenhuma correlação significativa foi identificada.

Ao investigar a correlação entre a $\mathrm{CF}$ e a recuperação da ventilação pulmonar, não foram identificadas correlações significativas para nenhuma variável analisada, tanto considerando todo o grupo ( $\mathrm{n}=22$, r sempre menor que 0,23$)$, quanto considerando os subgrupos analisados: EI ( $n=16, r$ sempre menor que 0,32$)$ e $E N(n=6, r$ sempre menor que 0,49$)$.

\section{Discussão}

O foco do presente estudo foi verificar a recuperação das variáveis cardiopulmonares $\mathrm{VO}_{2}$ e VE após esforço progressivo máximo de atletas de RCR, buscando verificar se o nível competitivo e a classificação funcional estavam associados à magnitude dessa recuperação. Os principais achados foram que os atletas de elite internacional apresentaram maior capacidade de recuperação de $\mathrm{VO}_{2}$ e $\mathrm{VE}$ nos primeiros minutos após o esforço e tal variação parece independer da CF nesse grupo. Para os atletas de elite nacional, que obtiveram menores valores de recuperação, a CF mostrou correlação forte e muito forte com a magnitude de recuperação de $\mathrm{VO}_{2}$. Tal fato pode ter uma associação com o tempo de treinamento, que é superior no primeiro grupo, indicando que o volume de prática semanal e tempo de prática da modalidade induzem a uma maior capacidade de recuperação cardiopulmonar. Adicionalmente, os resultados sugerem que o tempo de treinamento pode minimizar a influência da $\mathrm{CF}$ na capacidade de recuperação de um atleta, uma vez que, no grupo de EI, piores valores de CF não estavam associados a piores valores de recuperação, fato observado em atletas do EN.

O treinamento físico induz adaptações crônicas em diferentes órgãos e sistemas, incluindo o cardiorrespiratório, que podem justificar as melhores respostas apresentadas pelo EI. Dentre elas, podem-se destacar modificações conhecidas da literatura clássica da fisiologia do treinamento no sistema vascular, advindos de maior concentração de substâncias vasodilatadoras, menor quantidade de estímulos simpáticos constritores ${ }^{18}$ e maior capilarização periférica $^{20}$, que promovem uma melhor irrigação muscular. Considerando que, durante a fase pós-esforço, o sistema cardiovascular se encontra modificado para atender às necessidades de recuperação desse organismo $^{21}$, as modificações crônicas previamente citadas poderão influenciar positivamente a recuperação e explicar os maiores valores da variação pico- 
recuperação em atletas com maior histórico e volume de treinamento. Cabe ressaltar que, mesmo que algumas comparações não tenham apresentado diferença estatística entre os grupos EN e EI, há uma clara tendência para valores maiores no grupo EI (elite internacional), cuja rotina de treinamento era mais volumosa. Talvez o pequeno tamanho amostral não tenha permitido a confirmação de diferenças estatísticas para todas as variáveis. Entretanto, olhando os resultados como um todo, incluindo a análise da recuperação absoluta e as correlações positivas do volume semanal com as variáveis cardiopulmonares, especialmente com a recuperação do $\mathrm{VO}_{2}$, parece que o nível competitivo, de fato, colabora para uma melhor recuperação cardiopulmonar após esforço máximo.

Sobre as adaptações fisiológicas ao exercício especificamente em indivíduos com lesão medular, tipo de lesão com uma grande representatividade entre os atletas da modalidade, West et $a l .{ }^{5}$ sugerem que o treinamento físico tem capacidade de atenuar as limitações cardiopulmonares provenientes das lesões nessa população. Sabe-se que, nessa população específica, o nível da lesão é inversamente proporcional à capacidade de resposta cardiorrespiratória, sendo uma das explicações a deficiência circulatória periférica advinda pelos comprometimentos neuromusculares e hipomobilidade dos membros ${ }^{22}$.

Retomando os resultados do presente estudo, a correlação da CF com a recuperação cardiopulmonar mostrou-se forte (e muito forte) no grupo que treinava menos e regular no grupo que treinava mais, podendo ser esse resultado uma evidência de que existe uma tendência à diminuição desta correlação conforme o tempo e volume de prática do atleta.

Uma pesquisa realizada por Flores et al. ${ }^{16}$, na qual foi avaliada a potência aeróbica de 10 atletas de RCR através de um teste de esforço submáximo de 12 minutos, correlacionando os valores obtidos do $\mathrm{VO}_{2 \text { máx }}$ com a classificação funcional e analisando o comportamento da frequência cardíaca, foi observado que existe uma correlação moderada $(r=0,77)$ entre a CF e o $\mathrm{VO}_{2 \text { máx }}$ e valores baixos de $\mathrm{FC}$ frente a um esforço submáximo. O volume semanal de treino dos atletas analisados (4,5h por semana) nesse estudo de Flores et al. ${ }^{16}$ se assemelham aos do grupo de atletas que treinavam menos do presente estudo (EN) e que apresentaram os níveis de recuperação menor. Tal nível de condicionamento (em ambos estudos) poderia explicar a forte correlação da CF encontrada no EN com a recuperação do consumo de oxigênio e da ventilação pulmonar.

No estudo de Molik et al. ${ }^{23}$ com a seleção feminina de basquete em cadeira de rodas do Canadá, constatou-se que havia uma correlação entre a classificação funcional das atletas e a 
performance anaeróbica, resultado que difere do encontrado no presente estudo. Cabe ressaltar que a modalidade estudada por esse grupo de autores abrange deficiências de menor impacto funcional que o RCR e que a variável desfecho considerada estava relacionada à potência anaeróbica e não à aeróbica, foco do presente estudo.

Devido à particularidade funcional de cada tipo de lesão medular e comprometimento motor, esse estudo foi limitado pelo tamanho da amostra, na qual poucos indivíduos possuíam o mesmo tipo de lesão, ainda que todos fossem tetraplégicos ou tetraequivalentes. A análise em caráter seccional limitou o acompanhamento da evolução das variáveis cardiopulmonares dos atletas, o que poderia confirmar a tendência de minimizar a CF como diferenciador do grau de recuperação dessas variáveis. Apesar das limitações, esse estudo conseguiu evidenciar aspectos relevantes inerentes ao comportamento da recuperação cardiopulmonar após esforço nessa população, demonstrando que o volume de prática da modalidade tem sido crucial na melhora dessa valência, com tendências a não depender do tipo de lesão e da classificação funcional. Sugere-se que novos estudos acrescentem análises de outras variáveis cardiopulmonares para um entendimento mais completo da capacidade de recuperação cardiopulmonar nesse público, uma vez que o presente estudo buscou analisar apenas as variáveis consumo de oxigênio e ventilação pulmonar, pela associação com nível de condicionamento físico da primeira e pelo impacto da tetraplegia na segunda. Somar novas variáveis e um tamanho amostral mais amplo poderá auxiliar no entendimento mais completo do fenômeno em atletas de Rugby em Cadeira de Rodas.

\section{Conclusão}

Os resultados observados como um todo demonstraram que o EI possui uma maior recuperação do consumo de oxigênio e da ventilação pulmonar nos três minutos subsequentes ao esforço quando comparado ao EN. Tal resultado indica que um maior volume de prática do RCR parece estar envolvido na melhor capacidade de recuperação cardiopulmonar para essa população. Cabe destacar que, como o tempo de lesão e a classificação funcional foram similares entre os grupos, tais variáveis não foram estatisticamente significativas a ponto de interferir nesses resultados. Quando o consumo de oxigênio e a ventilação pulmonar foram correlacionados à $\mathrm{CF}$, observou-se no $\mathrm{EN}$ uma forte correlação com o consumo de oxigênio. No entanto, ao analisarmos essa mesma correlação no grupo EI, ela não se fez presente, o que 
parece indicar que o treinamento minimiza a influência da CF na capacidade de recuperação cardiopulmonar pós-esforço de alta intensidade.

Dessa forma, as comissões técnicas das equipes de RCR podem utilizar uma nova medida fisiológica importante tanto para acompanhar a eficácia de um treinamento, quanto para identificar que atletas precisam de mais tempo para se recuperar durante os treinos e jogos: a recuperação do $\mathrm{VO}_{2}$ e da $\mathrm{VE}$ pós-esforço progressivo de alta intensidade. Estudos futuros devem analisar essas variáveis de forma longitudinal, buscando investigar as relações entre o período pré-treinamento e o pós-treinamento, analisando a evolução dos indivíduos, em especial através de ensaios clínicos randomizados, aplicando a prática do RCR como intervenção em diferentes volumes semanais.

\section{Agradecimentos}

O estudo recebeu suporte da Fundação Carlos Chagas Filho de Amparo à Pesquisa do Estado do Rio de Janeiro (FAPERJ), edital "Programa apoio ao desenvolvimento de inovações no esporte no estado do Rio de Janeiro - 2013” (número do processo: 190.228/2013). Os autores também agradecem à Academia Paralímpica Brasileira, do Comitê Paralímpico Brasileiro $(\mathrm{APB} / \mathrm{CPB})$, pelo apoio científico, bem como às equipes participantes do estudo.

\section{Referências}

1. IWRF - International Wheelchair Rugby Federation. Introduction to Wheelchair Rugby. 2012. Disponível em: <http://www.iwrf.com/resources/iwrf_docs/Introduction-toWheelchair-Rugby-2012.pdf $>$. Acesso em: 8 maio 2015.

2. IWRF - International Wheelchair Rugby Federation. A Laypersons Guide to Wheelchair Rugby Classification. 2015. Disponível em:

<http://www.iwrf.com/resources/iwrf_docs/Laypersons_Guide_Classification.pdf>. Acesso em: 30 maio 2015.

3. Barfield JP, Malone LA, Arbo C, Jung AP. Exercise Intensity During Wheelchair Rugby Training. J Sports Sci. 2010; 28(4): 389-98.

4. Rhodes JM, Mason BS, Malone LA, Goosey-Tolfrey VL. Effect of Team Rank and Player Classification on Activity Profiles of Elite Wheelchair Rugby Players. J Sports Sci. 2015; 33(19): 2070-8.

5. West CR, Campbell IG, Shave RE, Romer LM. Resting Cardiopulmonary Function in Paralympic Athletes with Cervical Spinal Cord Injury. Med Sci Sports Exerc. 2012; 44(2): 323-9. 
6. Balady GJ, Arena R, Sietsema K, Myers J, Coke L, Fletcher GF et al. Clinician's Guide to Cardiopulmonary Exercise Testing in Adults: A Scientific Statement From the American Heart Association. Circulation. 2010; 122(2):191-225.

7. ACSM - American College of Sports Medicine. ACSM's Guidelines for Exercise Testing and Prescription, 9th ed, Philadelphia: Wolters Kluwer/ippincott Williams \& Wilkins Health, 2013.

8. Campos LFCC. Comparação entre métodos para mensuração da potência aeróbia em atletas tetraplégicos. [Dissertação de Mestrado]. Campinas: Programa de Pós-Graduação da Faculdade de Educação Física, UNICAMP; 2013.

9. Guazzi M, Adams V, Conraads V, Halle M, Mezzani A, Vanhees L, et al. Clinical recommendations for cardiopulmonary exercise testing data assessment in specific patient populations. Eur Heart J. 2012; 33(23): 2917-27.

10. Goosey-Tolfrey VL, Castle P, Webborn N. Aerobic Capacity and Peak Power Output of Elite Quadriplegic Games Players. Br J Sports Med. 2006; 40(8): 684-7.

11. Goosey-Tolfrey VL, Leicht C, Lenton J, Diaper N, Mason B. The BASES Expert Statement on Assessment of Exercise Performance in Athletes with a Spinal Cord Injury. TSES. 2013; 37(2): 8-9.

12. Janssen TW, Dallmeijer AJ, Veeger DJ, Van der Woude LH. Normative values and determinants of physical capacity in individuals with spinal cord injury. 2002. J Rehabil Res Dev. 2002; 39(12): 29-39.

13. IWRF - International Wheelchair Rugby Federation. International Rules for the Sport of Wheelchair Rugby.2015. Disponível em: <http://www.iwrf.com/resources/iwrf_docs /Wheelchair_Rugby_International_Rules_2015_English.pdf>. Acesso em: 30 abr. 2015.

14. Simões RP, Bonjorno Jr JC, Beltrame T, Catai AM, Arena R, Broghi-Silva A. Slower heart rate and oxygen consumption kinetic responses in the on- and off-transient during a discontinuous incremental exercise: effects of aging. Braz J Phys Ther. 2013; 17(1): 69-76.

15. Baumgart JK, Brurok B, Sandbakk Ø. Peak oxygen uptake in Paralympic sitting sports: A systematic literature review, meta- and pooled-data analysis. PLoS ONE. 2018; 13(2): e0192903.

16. Flores LJF, Gorla JI, Campos LFCC, Gouveia RB, Silva AAC, Pena LGS. Avaliação da Potência aeróbia de praticantes de Rugby em Cadeira de Rodas através de um teste de quadra. Motriz. 2013; 19(2): 368-77.

17. Taskin H, Erkmen N, Cicioglu I. The Examination of the Heart Rate Recovery after Anaeróbic Running in Soccer Players. Coll Antropol. 2014; 38(1): 207-11.

18. ACSM - American College of Sports Medicine. Exercise and Hypertension - Position Stand. Med Sci Sports Exerc. 2004; 36(3): 533-53. 
19. Callegari-Jacques, SM: Bioestatística: princípios e aplicações. Porto Alegre: Artemed, 2003.

20. Fagard RH. Exercise characteristics and the blood pressure response to dynamic physical training. Med Sci Sports Exerc. 2001; 33(6): 484-92.

21. Romero SA, Minson CT, Halliwill JR. The cardiovascular system after exercise. J Appl Physiol. 2017; 122(4): 925-32.

22. Menezes-Reis R, Ribeiro VB, Tourinho HF. Respostas Fisiológicas ao Exercício Físico em Atletas Cadeirantes com Lesão Medular. Conscientiae saúde. 2015; 14(1): 161-8.

23. Molik B, Laskin JJ, Kosmol A, Marszalek J, Morgulec N, Frick T. Relationships Between Anaerobic Performance, Field Tests, and Functional Level of Elite Female Wheelchair Basketball Athletes. Hum Mov. 2013; 14(4): 366-71. 